\title{
Sufficient Conditions of Asymptotic Stability of the Time-Varying Descriptor Systems
}

\author{
Xiaoming Su and Yali Zhi \\ School of Science, Shenyang University of Technology, Shenyang 110870, China \\ Correspondence should be addressed to Yali Zhi; zhiyali87828@163.com \\ Received 20 June 2013; Revised 10 October 2013; Accepted 6 November 2013 \\ Academic Editor: Shuli Sun
}

Copyright (C) 2013 X. Su and Y. Zhi. This is an open access article distributed under the Creative Commons Attribution License, which permits unrestricted use, distribution, and reproduction in any medium, provided the original work is properly cited.

\begin{abstract}
We discuss the time-varying descriptor systems. Firstly, a sufficient condition of asymptotic stability and impulse-free is derived based on Riccati equation. Secondly, we design a state feedback controller to make the close-loop system asymptotically stable and impulse-free. Finally, a numerical example demonstrates the proposed results.
\end{abstract}

\section{Introduction}

Descriptor systems, also known as generalized state systems, have been widely studied in the past several decades. Descriptor systems have some special properties, such as regularity and impulse behavior. Asymptotic stability is one of the properties for this control systems. Many researchers have focused on the problems of asymptotic stability.

Lyapunov approach plays an important role in the analysis of control systems. Lyapunov approach has become a powerful method for standard state space systems. In recent years, many researchers are trying to apply this approach to descriptor systems.

Lyapunov equation of descriptor systems were firstly presented by Campell and Petzold [1], and the results were promoted by Lewis [2]; Lyapunov equation was used to study the structural stability of the descriptor systems by Khargonekar et al. [3], Wei [4], and Takaba et al. [5]; Lyapunov inequality and Riccati equation are introduced by Wei [4] and Takaba et al. [5]; Hsiung and Lee [6]; [7-10] presented necessary and sufficient conditions of the stability for timevarying descriptor systems; in [11], admissibility analysis for time-varying periodically descriptor systems was given; [12] presents new results for a generalized Lyapunov approach of linear time-varying periodically descriptor systems; a linear matrix differential inequality approach of linear descriptor systems was obtained in [13]; [14-17] discussed the stabilization of several class of descriptor systems. Up to now, scholars introduced the necessary and sufficient condition of asymptotic stability and used the Lyapunov equation for solving the problems of asymptotic stability, stabilization; there is no effective method for solving the stabilization via a Riccati equation.

In this paper, we mainly consider sufficient conditions of asymptotic stability and impulse-free based on Riccati equation; the fast subsystem can be determined by the slow subsystem according to the second limited equivalence transformation on the previous basis; then we give a state feedback controller to make the time-varying descriptor systems asymptotically stable and impulse-free.

The paper is organized as follows. Section 2 presented the sufficient condition of asymptotic stability and impulse-free for the time-varying descriptor system. Section 3 gives the design of the state feedback controller of the time-varying descriptor system based on Lyapunov and Riccati equations. In Section 4, a numerical example illustrates the content of this paper studies in-line with the actual demand; the method also is more simple.

\section{Asymptotic Stability of Time-Varying Descriptor Systems}

Consider the following time-varying descriptor system:

$$
E(t) \dot{x}(t)=A(t) x(t)+B(t) u(t),
$$


where $x(t) \in R^{n}$ is the state and $y(t) \in R^{q}$ is the output. $A(\cdot), B(\cdot)$ is analytic matrix function. $E(t) \in R^{n}$ and $\operatorname{rank} E(t)=q<n$.

Definition 1 (see [9]). For system (1), if there exists a scalar $s$, such that $\operatorname{det}(s E(t) A(t)) \neq 0$, for all $t \in[0, \infty)$, then the system (1) is called uniformly regular.

From Definition 1, the regularity of the system (1) is equivalent to the analytical solvability in the sense of Campbell [7]. Put the system into the following decomposition.

For system (1), suppose the constant invertible matrices $P$ and $Q$ such that

$$
\begin{gathered}
P E(t) Q=\left[\begin{array}{cc}
I(t) & 0 \\
0 & 0
\end{array}\right], \quad P A(t) Q=\left[\begin{array}{ll}
A_{11}(t) & A_{12}(t) \\
A_{21}(t) & A_{22}(t)
\end{array}\right], \\
P B(t)=\left[\begin{array}{l}
B_{1}(t) \\
B_{2}(t)
\end{array}\right], \quad Q^{-1} x(t)=\left[\begin{array}{l}
x_{1}(t) \\
x_{2}(t)
\end{array}\right]
\end{gathered}
$$

then the system (1) is restricted equivalent to

$$
\begin{aligned}
& \dot{x}_{1}(t)=A_{11}(t) x_{1}(t)+A_{12}(t) x_{2}(t)+B_{1}(t) u(t), \\
& 0=A_{21}(t) x_{1}(t)+A_{22}(t) x_{2}(t)+B_{2}(t) u(t) .
\end{aligned}
$$

Obviously, the necessary and sufficient condition of impulsefree a for the system (1) is that $A_{22}(t)$ is invertible.

Definition 2 (see [10]). If for any initial state: $x(0) \in R^{n}$, there exist real number $\alpha, \beta>0$ such that $\|x(t)\|_{2} \leq \alpha e^{-\beta t}\|x(0)\|_{2}$, then the system (1) is called asymptotically stable.

Lemma 3 (see [10]). If $x, y \in R^{n}, A, B \in R^{n \times n}$, then for any for all $\varepsilon>0,2 x^{T} A B y \leq \varepsilon x^{T} A A^{T} x+(1 / \varepsilon) y^{T} B^{T} B y$. When $u(t)=0$, system (1) can be rewritten as follows:

$$
E(t) \dot{x}(t)=A(t) x(t) .
$$

Then, we have

$$
\dot{x}_{1}(t)=\left(A_{11}(t)-A_{12}(t) A_{22}^{-1}(t) A_{21}(t) x_{1}(t)\right) .
$$

Theorem 4. Time-varying descriptor systems (4) is said asymptotically stable and impulse-free, if for all $t \in[0, T]$; system (4) is uniformly regular, and the following conditions hold at the same time:

(1) $\|A(t)\| \leq m, m>0$;

(2) $A_{22}(t)$ is invertible;

(3) if there exist $\varepsilon>0, Q$ is a symmetric matrix, so that Riccati equation

$$
A_{11}^{T}(t) P+P A_{11}(t)+\frac{1}{\varepsilon} P^{T} P+\varepsilon b^{2} m^{4} I+\frac{1}{\varepsilon} Q=0
$$

has a positive definite symmetric solution $P$, where $b=$ $\sup \left\|A_{22}^{-1}(t)\right\|$.
Proof. Since system (4) is uniformly regular, all for all $t \geq$ $0, A_{22}(t)$ is invertible; then system (4) is impulse-free.

Let $V=x_{1}(t)^{T} P x_{1}(t) ; P$ is a symmetric matrix,

$$
\begin{aligned}
\dot{V}= & \frac{d\left(x_{1}^{T}(t) P x_{1}(t)\right)}{d t} \\
= & \dot{x}_{1}^{T}(t) P x_{1}(t)+x_{1}^{T}(t) P \dot{x}_{1}(t) \\
= & {\left[x_{1}(t)^{T} A_{11}(t)^{T}+x_{2}(t)^{T} A_{12}(t)^{T}\right] P x_{1}(t) } \\
& +x_{1}(t)^{T} P\left[A_{11}(t) x_{1}(t)+A_{12}(t) x_{2}(t)\right] \\
= & x_{1}(t)^{T}\left[A_{11}(t)^{T} P+P A_{11}(t)\right] x_{1}(t) \\
& +x_{1}(t)^{T} P A_{12}(t) x_{2}(t)+x_{2}(t)^{T} A_{12}(t)^{T} P x_{1}(t) \\
= & x_{1}(t)^{T}\left[A_{11}(t)^{T} P+P A_{11}(t)\right] x_{1}(t) \\
& +2 x_{2}(t)^{T} A_{12}(t)^{T} P x_{1}(t) ;
\end{aligned}
$$

by Lemma 3, we get

$$
\begin{aligned}
\dot{V} \leq & x_{1}(t)^{T}\left[A_{11}(t)^{T} P+P A_{11}(t)\right] x_{1}(t) \\
& +\varepsilon x_{2}(t)^{T} A_{12}(t)^{T} A_{12}(t) x_{2}(t)+\frac{1}{\varepsilon} x_{1}(t)^{T} P^{T} P x_{1}(t),
\end{aligned}
$$

since

$$
\begin{aligned}
\left\|x_{2}(t)\right\| & =\left\|-A_{22}^{-1}(t) A_{21}(t) x_{1}(t)\right\| \\
& \leq \sup \left\|A_{22}^{-1}(t)\right\| \cdot\left\|A_{21}(t)\right\| \cdot\left\|x_{1}(t)\right\| \\
& \leq m \sup \left\|A_{22}^{-1}(t)\right\| \cdot\left\|x_{1}(t)\right\| \\
& =m b\left\|x_{1}(t)\right\|,
\end{aligned}
$$

so

$$
\dot{V} \leq x_{1}(t)^{T}\left[A_{11}(t)^{T} P+P A_{11}(t)+\varepsilon m^{4} b^{2} I+\frac{1}{\varepsilon} P^{T} P\right] x_{1}(t),
$$

by the condition 3 , we get

$$
\begin{aligned}
\dot{V} & \leq \frac{1}{\varepsilon} x_{1}(t)^{T}(-Q) x_{1}(t)=-\frac{1}{\varepsilon} x_{1}(t)^{T}(Q) x_{1}(t) \\
& \leq \frac{1}{\varepsilon} \sigma_{\min }(Q) \cdot\left\|x_{1}(t)\right\|^{2},
\end{aligned}
$$

where $\sigma_{\min }(Q)$ is $Q$ minimum eigenvalue, according to Lyapunov stability; we have $x_{1}(t)$ is asymptotically stable, since $x_{2}(t)$ can be determined by $x_{1}(t)$.

Then time-varying descriptor systems (4) is said asymptotically stable.

The proof is completed. 


\section{Asymptotic Stabilization of Time-Varying Descriptor Systems}

Definition 5 (see [12]). The system (1) is called strongly asymptotically stable, if the system (1) is impulse-free and asymptotical stable.

Definition 6 (see [16]). For the system (1), if there exists a state feedback $u(k)=-K(t) x(t)$, such that the close-loop system

$$
E(t) \dot{x}(t)=(A(t)-B(t) K(t)) x(t)
$$

is strongly asymptotically stable, then the system (1) is called stabilized.

Theorem 7. Time-varying descriptor system (1) is said to be stabilized, if for all $t \in[0, T]$; system (12) is uniformly regular, and the following conditions hold at the same time:

(1) $\|\bar{A}(t)\| \leq m, m>0$;

(2) for all $t \geq 0, A_{22}(t)$ is invertible;

(3) if there exist $\varepsilon>0, Q$ is a symmetric matrix, so that Riccati equation,

$$
\left[\begin{array}{ccc}
\varepsilon\left(A_{11}(t)^{T}-K_{1}(t)^{T} B_{1}(t)^{T}\right) P & P\left(A_{11}(t)-B_{1}(t) K_{1}(t)\right) & P^{T} \\
I & -\frac{1}{\varepsilon} I & 0 \\
P & 0 & -I
\end{array}\right]
$$$$
+\varepsilon b^{2} m^{2} I+\frac{1}{3} Q=0
$$

has a positive definite symmetric solution $P$, and there exists a state feedback

$$
K(t)=\left[\begin{array}{ll}
K_{1}(t) & 0
\end{array}\right], \quad K_{1}(t)=-\frac{1}{\varepsilon} B_{1}^{-1}(t) P,
$$

where $\bar{A}(t)=A(t)-B(t) K(t), \bar{A}_{22}(t)=A_{22}(t)$, and $b=$ $\sup \left\|\bar{A}_{22}^{-1}(t)\right\| \cdot\left\|B_{2}(t) K_{1}(t)-A_{21}(t)\right\|$.

Proof. By Schur complement, it is easy to check that inequality (13) holds if and only if the following inequality holds:

$$
\begin{aligned}
& \left(A_{11}^{T}(t)-K_{1}(t)^{T} B_{1}(t)^{T}\right) P \\
& \quad+P\left(A_{11}(t)-B_{1}(t) K_{1}(t)\right)+\frac{1}{\varepsilon} P^{T} P \\
& \quad+\varepsilon b^{2} m^{2} I+\frac{1}{3} Q=0 .
\end{aligned}
$$

Taking the same line as Theorem 4, proved.

\section{Numerical Example}

For time-varying descriptor system (4), when $0<t \leq 1$,

$$
E=\left[\begin{array}{ccc}
t & 0 & 0 \\
0 & t & 0 \\
0 & 0 & 0
\end{array}\right], \quad A=\left[\begin{array}{ccc}
0 & 1 & 0 \\
2 & 0 & 2 \\
0 & 0 & -t
\end{array}\right]
$$

It is easy to calculate $0<\|A(t)\|=2 t \leq 2,\left\|A_{22}(t)^{T}\right\|=t>$ $0,0<t \leq 1$; obviously, the system (4) is uniformly regular and impulse-free; let $m=2, b=1, \varepsilon=0.1$, and $Q=\left[\begin{array}{ll}1 & 0 \\ 0 & 1\end{array}\right]$, by the Riccati equation,

$$
A_{11}^{T}(t) P+P A_{11}(t)+\frac{1}{\varepsilon} P^{T} P+\varepsilon b^{2} m^{4} I+\frac{1}{\varepsilon} Q=0 ;
$$

we get a positive definite symmetric solution $P$, and $P=$ $\left[\begin{array}{ll}1.0943 & 0.1497 \\ 0.1497 & 1.0805\end{array}\right]$, so the system (4) is asymptotically stable and impulse-free.

\section{Conclusions}

In this paper, a sufficient condition of asymptotical stability and impulse-free was presented, and a state feedback controller was designed in terms of Riccati equation to make the time-varying descriptor system asymptotically stable and impulse-free. Finally, a numerical example was presented to illustrate the main results.

\section{Acknowledgments}

This project is supported by the National Nature Science Foundation of China (no. 61074005) and the Talent Project of the High Education of Liaoning province, China, under Grant no. LR2012005.

\section{References}

[1] L. S. Campell and L. R. Petzold, "Canonical forms and solvable singular systems of differential equations," SIAM Journal on Algebraic and Discrete Methods, vol. 4, no. 4, pp. 517-521, 1983.

[2] F. L. Lewis, "A survey of linear singular systems," Circuits, Systems, and Signal Processing, vol. 5, no. 1, pp. 3-36, 1986.

[3] P. P. Khargonekar, I. R. Petersen, and K. Zhou, "Robust stabilization of uncertain linear systems: quadratic stabilizability and $H_{\infty}$ control theory," IEEE Transactions on Automatic Control, vol. 35, no. 3, pp. 356-361, 1990.

[4] K. Wei, "Stabilization of linear time-invariant interval systems via constant state feedback control," IEEE Transactions on Automatic Control, vol. 39, no. 1, pp. 22-32, 1994.

[5] K. Takaba, N. Morihira, and T. Katayama, "A generalized Lyapunov theorem for descriptor system," Systems \& Control Letters, vol. 24, no. 1, pp. 49-51, 1995.

[6] K. L. Hsiung and L. Lee, "Lyapunov inequality and bounded real lemma for discrete-time descriptor systems," IEE Proceedings, vol. 146, no. 4, pp. 327-331, 1999.

[7] S. Hu and J. Wang, "On stabilization of a new class of linear time-invariant interval systems via constant state feedback control," IEEE Transactions on Automatic Control, vol. 45, no. 11, pp. 2106-2111, 2000.

[8] S. Bittati, "30 years of periodic control-from analysis to design," in Proceedings of the 3rd Asian Control Conference, pp. 1253-1258, Shanghai, China, 2000.

[9] X. Su and Q. Zhang, "Stability for time-varying singular system," Journal of Northeastern University, vol. 22, no. 5, pp. 575-578, 2001.

[10] G. Hu and J. Sun, "Stability analysis for singular systems with time-varying," Journal of Tongji University, vol. 31, no. 4, pp. 481485, 2003. 
[11] X. Su and M. Lv, "Analysis of admissibility for periodically timevarying descriptor systems," Journal of Shenyang University of Technology, vol. 27, no. 6, pp. 710-713, 2005.

[12] X. Su, M. Lv, H. Shi, and Q. Zhang, "Stability analysis for linear time-varying periodically descriptor systems: a generalized Lyapunov approach," in Proceedings of the 6th World Congress on Intelligent Control and Automation (WCICA '06), pp. 728732, Dalian, China, June 2006.

[13] M. Inoue, T. Wada, M. Ikeda, and E. Uezato, "Stabilization of linear time-varying descriptor systems: a linear matrix differential inequality approach," in Proceedings of the International Conference on Control, Automation and Systems (ICCAS '08), pp. 1855-1860, COEX, Seoul, Korea, October 2008.

[14] D. Wu and J. Zhu, "On stability for a class of time-varying singular system," Journal of Tongji University, vol. 38, no. 6, pp. 925934, 2010.

[15] W. H. Chen, W. Fu, R. Du, and X. Lu, "Exponential stability of a class of linear time-varying singularly perturbed systems," in Proceedings of the International Conference on Information Science and Technology (ICIST '11), pp. 778-783, Jiangsu, China, March 2011.

[16] X. Su, M. Lv, H. Shi, and Q. Zhang, "Stability analysis for linear time-varying periodically descriptor systems: a generalized Lyapunov approach," in Proceedings of the 6th World Congress on Intelligent Control and Automation (WCICA '06), pp. 728732, Dalian, China, June 2006.

[17] M. Inoue, T. Wada, M. Ikeda, and E. Uezato, "Stabilization of linear time-varying descriptor systems: a linear matrix differential inequality approach," in Proceedings of the International Conference on Control, Automation and Systems (ICCAS '08), pp. 1855-1860, COEX, Seoul, Korea, October 2008. 


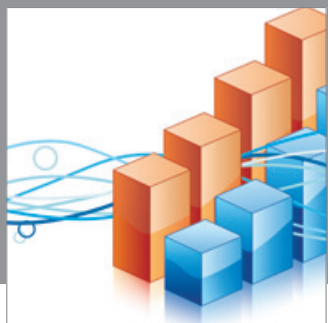

Advances in

Operations Research

mansans

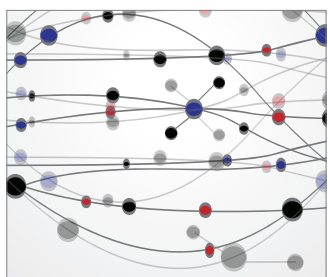

The Scientific World Journal
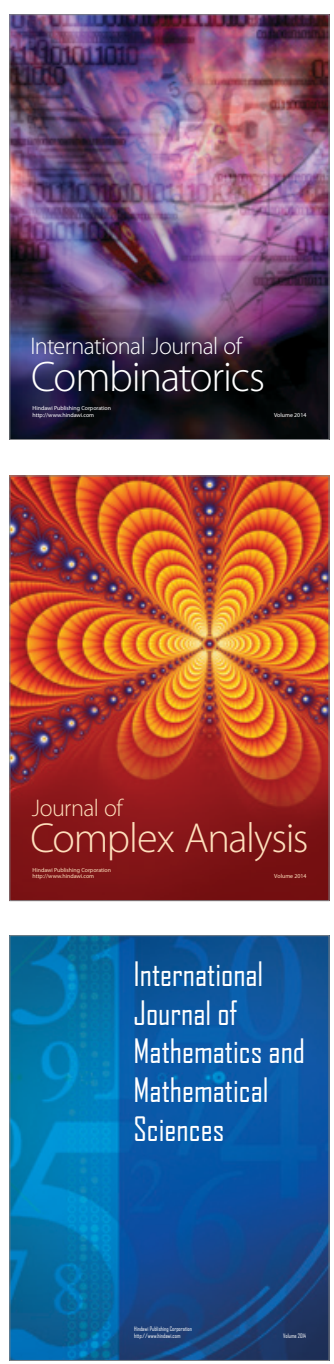
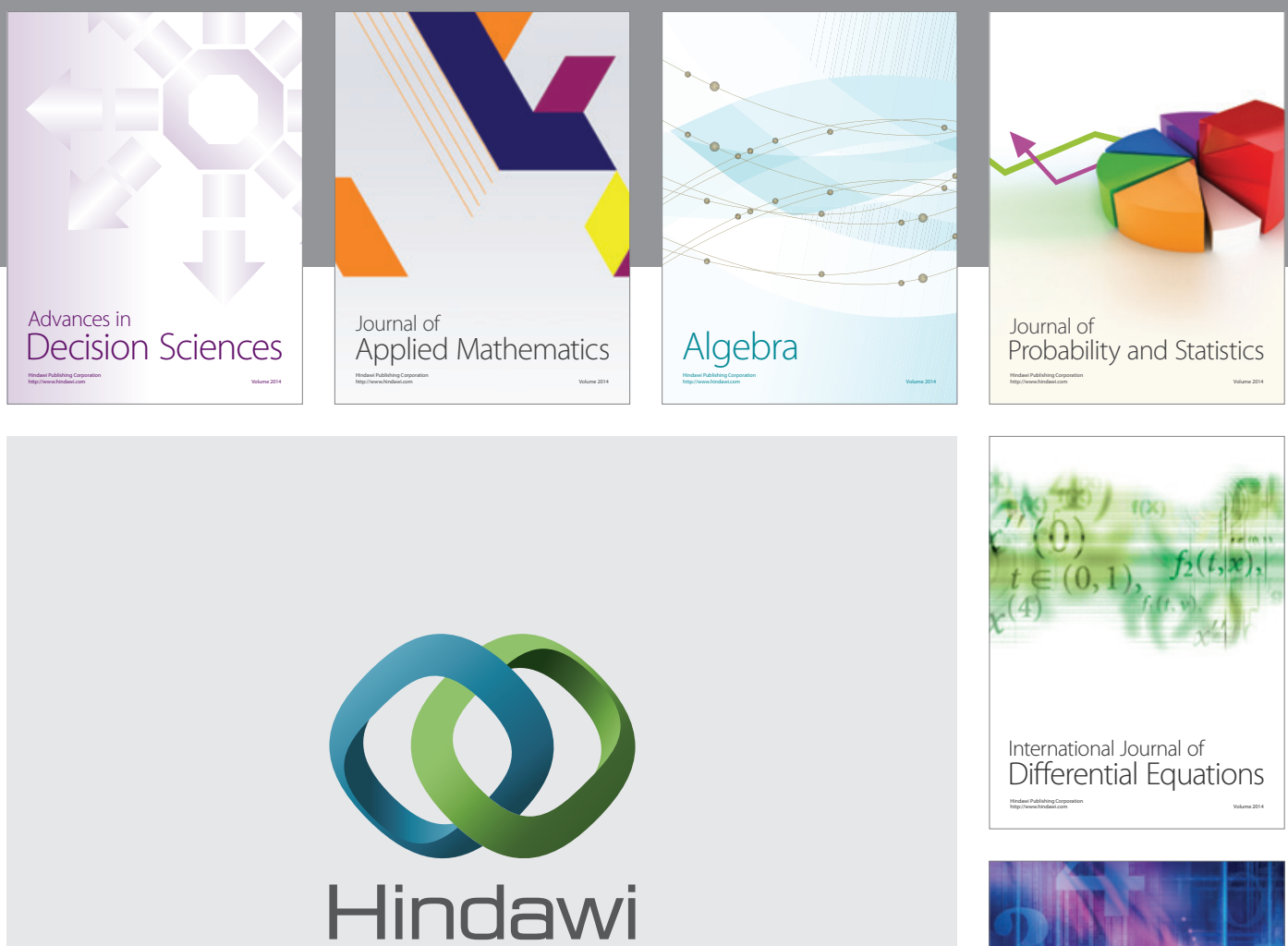

Submit your manuscripts at http://www.hindawi.com
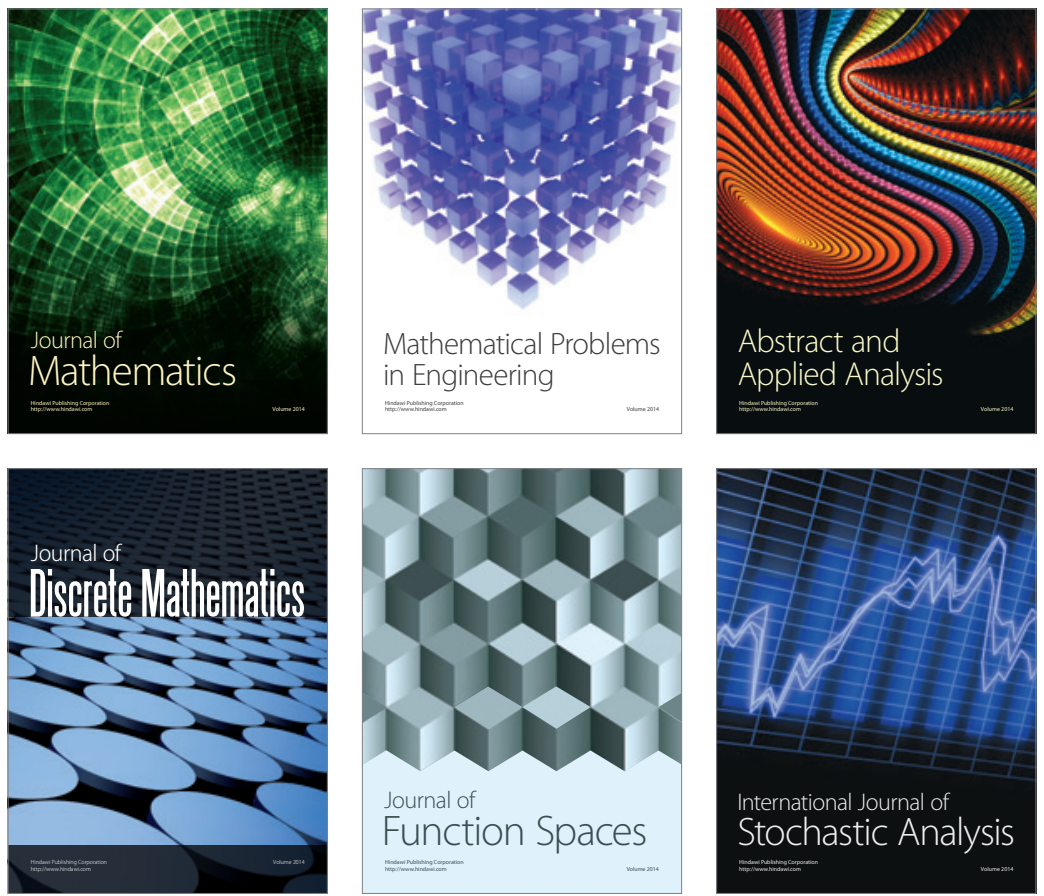

Journal of

Function Spaces

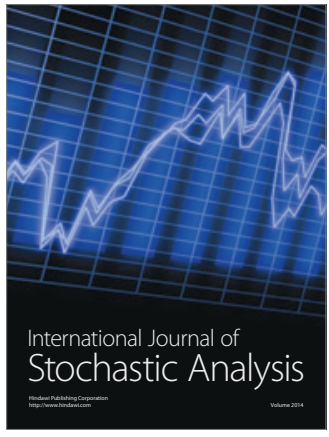

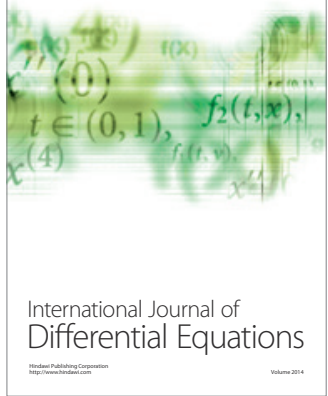
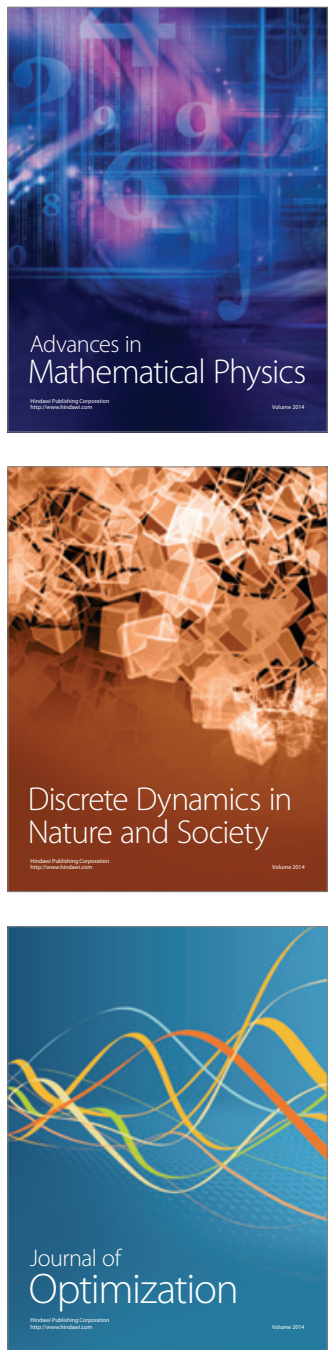